\title{
Category norms for 500 Spanish words in five semantic categories
}

\author{
CRISTINA IZURA \\ University of Wales, Swansea, Wales \\ NATIVIDAD HERNÁNDEZ-MUÑOZ \\ University of Salamanca, Salamanca, Spain \\ and \\ ANDREW W. ELLIS \\ University of York, York, England
}

\begin{abstract}
This study presents a database of 500 words from five semantic categories: animals, body parts, furniture, clothing, and intelligence. Each category contains 100 words, and data on lexical availability, age of acquisition, imageability, typicality, concept familiarity, written word frequency, and word length in number of syllables are provided with each word. The full set of norms may be downloaded from www.psychonomic.org/archive.
\end{abstract}

The study of semantic or natural categories became a central theme in cognitive research following the pioneering prototype theory proposed by Rosch (1973). This theory suggests that we group our knowledge of the world into semantic categories, the most prominent characteristic of which is that some members are better examples of a category than others are (e.g., a robin is a better example of the category birds than a penguin is). Since the formulation of this theory, a large amount of effort has been devoted to the study of the components and the organization of semantic categories in cognitive research (e.g., Casey, 1992; Laiacona, Capitani, \& Caramazza, 2003; Loftus \& Suppes, 1972; McFarland, Kellas, Klueger, \& Juola, 1974; Mervis, Catlin, \& Rosch, 1975) and neuropsychological research (e.g., Adrados, González, Sánchez, \& Galeote, 2001; Monsch et al., 1994; Tröster, Salmon, McCullough, \& Butters, 1989). In order to investigate the semantic world and its organization, a number of tasks have been used, such as the category generation task, in which participants have to generate as many members of a given category as possible, or the category verification task, in which participants are required to decide whether a stimulus belongs to a given category or not. The principal characteristic of these tasks is that in order

This research is part of the El léxico disponsible del hablante hispano: Aportación de datos y replanteamiento teórico project and was supported by Ministerio de Ciencia y Tecnología Grant BFF2001-1005. We thank Gerardo Aguado and Fernando Cuetos for giving us permission to use their Spanish dictionary of frequency norms. Correspondence concerning this article should be addressed to C. Izura, Department of Psychology, University of Wales, Singleton Park, Swansea SA2 8PP, Wales (e-mail: c.izura@swansea.ac.uk).

Note-This article was accepted by the previous editor, Jonathan Vaughn for them to be completed, participants need to access information about the meaning of the target words. That is, the tasks require semantic activation, and this activation is assumed to be reflected in the outcome of the tasks. However, they also involve the production or recognition of a verbal item, and as a consequence, investigations in semantic memory have to take into account the lexical and semantic properties of the experimental materials used.

The semantic property most widely studied in categorization research is typicality (Koivisto \& Laine, 1999; McFarland, Duncan, \& Kellas, 1978; Mervis \& Rosch, 1981; Smith, Shoben, \& Rips, 1974). Typicality refers to the degree to which a concept (e.g., $d o g$ ) is representative of a given category (e.g., animals). The frequency of occurrence of category names and its instances ("category dominance" and "instance dominance," respectively) are factors also taken into consideration from early studies (Loftus, 1973; McFarland et al., 1974). Recent studies, however, have revealed the influence of additional variables on semantic memory and/or on the formation of categories. For example, a number of studies have argued that age of acquisition (AoA) is an important factor influencing the speed of word recognition and production, with faster processing times for words acquired first or early in life than for words acquired some time later (Barry, Morrison, \& Ellis, 1997; Izura \& Ellis, 2002; Monaghan \& Ellis, 2002), and recent research has demonstrated that AoA affects performance in semantic tasks (Brysbaert, Van Wijnendaele, \& De Deyne, 2000; Izura $\&$ Ellis, 2004). Other variables, such as concept familiarity and imageability, have been reported to contribute to the performance of the lexico-semantic system with more effective or rapid processing of familiar and highly imageable concepts (Coltheart \& Winograd, 1986; Ellis \& Morrison, 1998; Mulligan, 1998). 
Norms for words organized into categories for adult speakers are scarce, and they provide a limited number of variables. In English, Battig and Montague (1969) and, in Spanish, Soto, Sabastián, García, and del Amo (1982) have reported measures of instance frequency and mean rank position. The present, study provides normative data for 500 words in five categories for the Spanish language. At present, there is no other study of these characteristics, in either the English or the Spanish language. Our motivation to elaborate the present norms arose from the experimental need for standardized material for words and categories. Our main aim now is to provide researchers in different fields (psychology, linguistics, and neuropsychology) with a useful tool with which to investigate the organization and processes of the lexico-semantic system. Whether this database is used for specific categorization studies or for more general language-processing research, the crucial aspect of the norms presented here is that they were collected in a category context, which means that all the variables have been rated within category boundaries. This is important if we consider the relation between word forms and meanings. These associations are not unambiguous. We find that different meanings can be expressed with a single word form (called polysemic words; e.g., present as a gift and as an indication of time), and we also find that different word forms can evoke the same meaning (e.g., sofa, settee). Measures collected out of a particular category context, such as randomly selected word lists, have insufficient meaning information. As a consequence, researchers are at risk of obtaining imprecise measures especially for polysemic words. For instance, the concept evoked by the Spanish word radio could be more typical, familiar, and acquired earlier as a sound device (English radio) than as a body part (English radius, a bone in the forearm). Typicality, familiarity, and AoA values might depend on the category, the idea, or the concept that the word is trying to convey. Thus, here we present norms for lexical research that are semantically more precise than other normative data collected out of category boundaries. These data will help researchers to design experiments more accurately.

The categories included in our norms are commonly used in cognitive, neuropsychological, and linguistic research. These categories are body parts, clothing, furniture, animals, and intelligence. The intelligence category was included in the study to act as a source of a more abstract vocabulary and, thus, provide researchers with material comprising a vocabulary with a wide range of abstract and concrete words. Our aim was accomplished, since intelligence was the category that elicited the higher number of low-imageable words ( $71 \%$ out of 100 words have an imageability value ranging from 2.52 to 4.48 ).

One hundred words were selected per category from the study by Hernández-Muñoz (2002), which is an exploration of the common vocabulary shared by the Spanish community of Cuenca. It comprises 5,873 different words organized in 11 categories and analyzed following different linguistic criteria, such as frequency of occur- rence and lexical availability (LA). The selection criterion for the present norms was based on LA values. LA refers to the ease with which a word can be accessed and produced as a member of a given category. The first 100 words with the highest LA scores in each category were selected for the present study. The computation procedure for LA values will be described below.

In a study of category fluency, based on the data presented in this article, Hernández-Muñoz, Izura, and Ellis (2005) found that LA, or the ease of production of instances of a given category in Spanish, is well predicted (56\% of the variance) by typicality, familiarity, and rated AoA, with a small contribution from written word frequency. This evidence is consistent with the established notion of typicality as a major factor determining categorization processes but also indicates the importance of other variables, such as concept familiarity and AoA, that might influence the performance of the lexicosemantic system.

The objective of this study was to cover the basic categories used in language research and to offer a complete range of variable values known to affect the structure and/or dynamics of the lexico-semantic system. Values on seven variables are reported: AoA, concept familiarity, typicality, imageability, LA, written word frequency, and word length.

\section{Database Variables}

The Appendix lists each word included in the database, along with typicality, concept familiarity, AoA, LA, imageability, word frequency, and word length.

Age of acquisition. Following the methodology used by Ghyselink, De Moor, and Brysbaert (2000), 50 monolingual native Spanish speakers with a mean age of 23 years (range, 18-42) were asked to indicate the age at which they believed they had first learned each word. This strategy was adopted as a less restricted and, therefore, more accurate measure of AoA than is the 5- or 7point scales commonly utilized in AoA studies, where late-acquired word ratings suffer the restriction of a limited scale. Every word was presented under its corresponding category label. The 500 words were divided into two sets of 250. Each set comprised 50 words from each of the five semantic categories and was completed by half the participants ( 25 per set). A number of researchers have reported a high correlation between AoA ratings and objective AoA measures, confirming the validity of AoA estimations (Carroll \& White, 1973; Gilhooly \& Gilhooly, 1980; Izura \& Ellis, 2002; Morrison, Chappell, \& Ellis, 1997).

Split-half intergroup reliability for these ratings was calculated by correlating scores (for the total 500 words) from half the participants (25) with scores from the other half (25). Reliability was .88. We also consulted Cuetos, Ellis, and Alvarez's (1999) Spanish norms for picture names as an extra assessment of the reliability of the AoA values presented here. Cuetos et al.'s norms were based on 144 pictures taken from Snodgrass and Van- 
derwart's (1980) set. They provided values for a set of variables, such as AoA, familiarity, word frequency, and so forth, and we found 55 words in common with the present study. The AoA values in the present study correlated highly $(r=.73)$ with Cuetos et al.'s AoA values.

Lexical availability. LA denotes the readiness with which a word is produced as a member of a given category. LA values are calculated from the results of category fluency tasks in which participants are asked to produce the highest number of instances of a particular category in a given time (e.g., $2 \mathrm{~min}$ ). An equation (see below) developed by López-Chávez and StrassburgerFrías (1987) was applied to the results of this task. The formula computes LA values, taking into consideration the position of the word in a given list/category for a given participant, the frequency of word production in a list/category, the total number of participants, and the lowest position in which a word has been produced across participants. The values resulting from the formula were less than zero and were multiplied by 100 for ease of presentation. The formula is

$$
D\left(P_{j}\right)=\sum_{i=1}^{n} e^{\left[-2.3 \times\left(\frac{i-1}{n-1}\right)\right]} \times \frac{f_{j i}}{I_{1}}
$$

where $D\left(P_{j}\right)$ is the LA of the word $j$ for a given category, $I$ is the total number of participants who completed the task, $i$ is the position of word $j$ in a given list, $f$ is the number of participants who wrote word $j$ at that position in their list, $n$ is the lowest position occupied by word $j$ in any list produced for the category in question, and $e$ is the natural number (2.718181818459045).

The LA values presented here were taken from Hernández-Muñoz (2002), who collected written category generation data from 117 Spanish native speakers 17 to 18 years of age.

Concept familiarity. Values were collected for the familiarity of the concept evoked by each of the 500 words. Ratings were gathered from a new group of 50 monolingual Spanish native speakers (mean age, 20 years; range, 17-53), using the same instructions (but translated) as those in Snodgrass and Vanderwart (1980). The participants were asked to estimate the degree to which they thought about or came into contact with a concept, using a 7-point scale (from $1=$ less than once a month to $7=$ many times a day). The 500 words were divided into sets of 250 (with 50 words per category in each set), each completed by half the participants.

Split-half intergroup reliability was .92. Reliability was also assessed by correlating the familiarity values presented here with Cuetos et al.'s (1999) reported familiarity for the 55 words found in both studies. The significant correlation $(r=.89)$ indicates that concept familiarity values are highly reliable.

Word frequency. Frequency values were taken from Alameda and Cuetos (1995), which is a corpus of written Spanish text comprising 2 million words. Concepts with more than one entry (singular and plural; masculine and feminine) were averaged in one value, and all frequency values were divided by 2 in order to present frequency per million words. Those words not included in Alameda and Cuetos's corpus were assigned a frequency of zero.

Typicality. Typicality ratings were collected from a third group of 50 Spanish native speakers (mean age $=$ 24 years; range, 18-36). The participants were divided into two groups, and each rated a set of 250 words, with 50 words per category. Instructions were adapted from Hampton and Gardiner (1983). The participants received an explanation on the meaning of typicality with some examples and were asked to rate each word on a scale ranging from 1 (very atypical example) to 7 (very typical example). Split-half intergroup reliability was .92.

Imageability. Ratings were obtained on a scale ranging from 1 (very difficult to arouse a mental image) to 7 (very easy to arouse a mental image; from Morrison et al., 1997). A final group of 50 monolingual native Spanish speakers (mean age $=23$ years; range, $17-58$ ) rated the 500 words presented in this study. The words were divided into two sets of 250 randomized words, with half the participants rating each set. Split-half intergroup reliability was .91 .

Word length. The number of syllables in a word is presented in these norms. Number of syllables was the word length measure of choice, since Cuetos et al. (1999) reported its influence on a picture-naming task.

\section{Results and Discussion}

One-way analyses of variance were used to compare the scores of the words in each category on each of the variables. There were significant differences among the categories on all of the predictor variables. These were analyzed further by using post hoc tests (Tukey's HSD) to compare the categories pairwise on each of the factors.

Age of acquisition. The categories differed significantly on AoA $\left[F(4,452)=8.03, M S_{\mathrm{e}}=35.61, p<.001\right]$. Items in the animals category were rated as earlier acquired than those in all the other categories, which did not differ significantly from each other on AoA.

Lexical availability. The categories differed overall on $\operatorname{LA}\left[F(4,452)=5.70, M S_{\mathrm{e}}=0.12, p<.001\right]$. Post hoc tests showed that the words from the intelligence category had significantly lower LAs than did the words from the other four categories, which did not differ significantly. This relates to the fact, noted above, that the words generated for the intelligence category were much more variable across participants than the words generated for the other categories. High variability means that individual words have been produced by fewer participants than words from the other categories. The formula for determining LA is such that smaller numbers of participants writing each word makes for smaller values of $f$ in the equation and, therefore, a lower value of LA.

Familiarity. The categories differed in the familiarity ratings given to the items within them $[F(4,452)=$ $\left.42.57, M S_{\mathrm{e}}=71.23, p<.001\right]$. Items in the intelligence category received the highest familiarity ratings, fol- 
lowed by furniture and body parts. Animals received the lowest familiarity ratings. Clothing items were intermediate between body parts and animals.

Word frequency. The categories differed significantly on word frequency $\left[F(4,452)=37.24, M S_{\mathrm{e}}=16.01, p<\right.$ $.001]$. Items in the intelligence category had the highest word frequencies, followed by body parts and furniture. Clothing and animals had the lowest frequencies.

Typicality. The categories differed significantly on typicality $\left[F(4,452)=9.72, M S_{\mathrm{e}}=16.85, p<.001\right]$. Items generated in the animals, body parts, and intelligence categories had the highest typicality ratings, whereas furniture and clothing had lower ratings. This implies that furniture and clothing are more widely dispersed categories in which items tend to stay further from the core of the category than is the case for animals, body parts, and intelligence.

Imageability. The categories differed significantly on imageability $\left[F(4,452)=155.34, M S_{\mathrm{e}}=90.44, p<\right.$ $.001]$. Items in the clothing, animals, and furniture categories were rated as most imageable. Body parts were less imageable, whereas items in the intelligence category had the lowest imageability ratings.

Word length. The categories differed significantly on word length $\left[F(4,452)=6.97, M S_{\mathrm{e}}=5.30, p<.001\right]$. Intelligence and furniture items had the longest names, whereas animals and body parts had the shortest. Clothing items were intermediate in length between furniture and animals.

\section{REFERENCES}

Adrados, H. P., González, M. J., Sánchez, M. L., \& Galeote, M. A. (2001). Batería de evaluación del deterioro de la memoria semántica en Alzheimer [Assessment battery for semantic memory deterioration in Alzheimer's]. Psychology in Spain, 5, 98-109.

Alameda, J. R., \& Cuetos, F. (1995). Diccionario de frecuencias de las unidas del castellano. Oviedo, Spain: Servicio de Publicaciones de la Universidad de Oviedo.

Barry, C., Morrison, C. M., \& Ellis, A. W. (1997). Naming the Snodgrass and Vanderwart pictures: Effects of age of acquisition, frequency and name agreement. Quarterly Journal of Experimental Psychology, 50A, 560-585.

Battig, A. F., \& Montague, W. E. (1969). Category norms for verbal items in 56 categories: A replication and extension of the Connecticut category norms. Journal of Experimental Psychology Monographs, 80, 1-45.

Brysbaert, M., Van Wijnendaele, I., \& De Deyne, S. (2000). Age of acquisition effects in semantic tasks. Acta Psychologica, 104, 215-226.

Carroll, J. B., \& White, M. N. (1973). Word frequency and age-ofacquisition as determiners of picture-naming latency. Quarterly Journal of Experimental Psychology, 25, 85-95.

CASEY, P. J. (1992). A reexamination of the roles of typicality and category dominance in verifying category membership. Journal of Experimental Psychology: Learning, Memory, \& Cognition, 18, 823-834.

Coltheart, V., \& Winograd, E. (1986). Word imagery but not age of acquisition affects episodic memory. Memory \& Cognition, 14, 174-180.

Cuetos, F., Ellis, A. W., \& Alvarez, B. (1999). Naming times for the Snodgrass and Vanderwart pictures in Spanish. Behavior Research Methods, Instruments, \& Computers, 31, 650-658.

Ellis, A. W., \& Morrison, C. M. (1998). Real age of acquisition effects in lexical retrieval. Journal of Experimental Psychology: Learning, Memory, \& Cognition, 24, 515-523.

Ghyselinck, M., De Moor, W., \& Brysbaert, M. (2000). Age-ofacquisition ratings for 2816 Dutch four- and five-letter nouns. Psychologica Belgica, 40, 77-98.
Gilhooly, K. J., \& Gilhooly, M. L. (1980). The validity of age-ofacquisition ratings. British Journal of Psychology, 71, 105-110.

Hampton, J. A., \& Gardiner, M. M. (1983). Measures of internal category structure: A correlational analysis of normative data. British Journal of Psychology, 74, 491-516.

HernándeZ-Muñoz, N. (2002). Disponibilidad léxica en Cuenca. Unpublished doctoral dissertation, Universidad de Salamanca.

Hernández-Muñoz, N., Izura, C., \& Ellis, A. W. (2005). Cognitive aspects of lexical availability. Manuscript submitted for publication.

IzUrA, C., \& Ellis, A. W. (2002). Age of acquisition effects in word recognition and production in first and second languages. Psicológica, 23, 245-281.

IzURA, C., \& Ellis, A. W. (2004). Age of acquisition effects in translation judgement tasks. Journal of Memory \& Language, 50, 165-181.

KoIvisto, M., \& LAINE, M. (1999). Strategies of semantic categorization in the cerebral hemispheres. Brain \& Language, 66, 341-357.

Laiacona, M., Capitani, E., \& Caramazza, A. (2003). Categoryspecific semantic deficits do not reflect the sensory/functional organization of the brain: A test of the "sensory quality" hypothesis. Neurocase, 9, 221-231.

LofTus, E. F. (1973). Category dominance, instance dominance and categorization time. Journal of Experimental Psychology, 97, 70-74.

Loftus, E. F., \& SupPES, P. (1972). Structural variables that determine the speed of retrieving words from long-term memory. Journal of Verbal Learning \& Verbal Behavior, 11, 770-777.

López Chávez, J., \& Strassburger Frías, C. (1987). Otro cálculo del índice de disponibilidad léxica: Presente y perspectiva de la investigación computacional en México. In H. López Morales (Ed.), Actas del IV simposio de la Asociación Mexicana de lingüística aplicada (pp. 187-207). Mexico City: UNAM.

McFarland, C. E., Jr., Duncan, E. M., \& Kellas, G. (1978). Isolating the typicality effect in semantic memory. Quarterly Journal of Experimental Psychology, 30, 251-262.

McFarland, C. E., Jr., Kellas, G., Klueger, K., \& Juola, J. (1974). Category similarity, instance dominance, and categorization time. Journal of Verbal Learning \& Verbal Behavior, 13, 698-708.

Mervis, C. B., Catlin, J., \& Rosch, E. (1975). Development of the structure of color categories. Developmental Psychology, 11, 54-60.

Mervis, C. B., \& Rosch, E. (1981). Categorization of natural objects. Annual Review of Psychology, 32, 89-115.

Monaghan, J., \& Ellis, A. W. (2002). What, exactly, interacts with spelling-sound consistency in word naming? Journal of Experimental Psychology: Learning, Memory, \& Cognition, 28, 183-206.

Monsch, A. U., Bondi, M. A., Butters, N., Paulsen, J. S., Salmon, D., Brugger, P., \& Swenson, M. R. (1994). A comparison of category deficit and letter fluency in Alzheimer's disease and Huntington's disease. Neuropsychology, 8, 25-30.

Morrison, C. M., Chappell, T. D., \& Ellis, A. W. (1997). Age of acquisition norms for a large set of object names and their relation to adult estimates and other variables. Quarterly Journal of Experimental Psychology, 50A, 528-559.

Mulligan, N. W. (1998). Perceptual interference at encoding enhances recall for high- but not low-imageability words. Psychonomic Bulletin \& Review, 5, 464-469.

Rosch, E. (1973). On the internal structure of perceptual and semantic categories. In T. E. Moore (Ed.), Cognitive development and the acquisition of language (pp. 111-144). New York: Academic Press.

Smith, E. E., Shoben, E. J., \& Rips, L. J. (1974). Structure and process in semantic memory: A feature model for semantic decisions. Psychological Review, 81, 214-241.

Snodgrass, J. G., \& VANDERWART, M. (1980). A standardized set of 260 pictures: Norms for name agreement, image agreement, familiarity, and visual complexity. Journal of Experimental Psychology: Human Learning \& Memory, 6, 174-215.

Soto, P., Sebastián, M. V., García, E., \& del Amo, T. (1982). Categorización y datos normativos en España (Colección Monografías, Instituto de Ciencias de la Educación, Universidad Autónoma de Madrid). Madrid: Ediciones Cantoblanco.

Tröster, A. I., Salmon, D. P., McCullough, D., \& Butters, N. (1989). A comparison of the category fluency deficits associated with Alzheimer's disease and Huntington's disease. Brain \& Language, 37, 500-513. 


\section{ARCHIVED MATERIALS}

The following materials and links may be accessed through the Psychonomic Society's Norms, Stimuli, and Data archive, http:// psychonomic. org/archive/.

To access these files or links, search the archive for this article using the journal (Behavior Research Methods), the first author's name (Izura), and the publication year (2005).

FILE: Izura-BRM-2005.zip

DESCRIPTION: izuranorms.xls, containing the norms developed by Izura, Hernández-Muñoz, and Ellis, as a $75 \mathrm{~K}$ tab-delimited text file generated by Excel 1997 for PCs. The five categories (animals, body parts, clothing, furniture, and intelligence) are represented in five different sheets. Each row represents one of 100 words per category. Each column represents one of the seven variables of interest (typicality, concept familiarity, age of acquisition, imageability, lexical availability, word frequency, and word length). The contents of izuranorms.xls are also available in csv format.

izuraaboutnorms.txt, a full description of the content of izuranorms.xls.

The Category Norms program may be used to select sets of stimuli comprising Spanish words belonging to five categories.

MAIN AUTHOR'S E-MAIL ADDRESS: c.izura@swansea.ac.uk.

\begin{tabular}{|c|c|c|c|c|c|c|c|}
\hline $\begin{array}{l}\text { Words Included in th } \\
\text { (Typ), Concept Fam } \\
\text { Lexical Availabil }\end{array}$ & (LA) & $\begin{array}{l}\text { A } \\
\text { Separs } \\
\text { am), A } \\
\text { ord Fr }\end{array}$ & $\begin{array}{l}\text { NDI } \\
\text { by C } \\
\text { f Acc } \\
\text { ency }\end{array}$ & $\begin{array}{l}\text { sory a } \\
\text { ition } \\
\text { areq), }\end{array}$ & $\begin{array}{l}\text { Their V: } \\
\text { A), Ima } \\
\text { d Word }\end{array}$ & $\begin{array}{l}\text { s in Ty } \\
\text { bility (I } \\
\text { gth (Le }\end{array}$ & \\
\hline $\begin{array}{c}\text { Spanish Word } \\
\text { (English Translation) }\end{array}$ & Typ & CFam & AoA & Imag & LA & WFreq & Len \\
\hline & & & mals & & & & \\
\hline Abeja (bee) & 4.28 & 3.56 & 4.48 & 6.76 & 7.672 & 4 & 3 \\
\hline Águila (eagle) & 4.84 & 3.24 & 5.24 & 6.80 & 19.365 & 15 & 3 \\
\hline Araña (spider) & 3.96 & 4.16 & 4.12 & 6.92 & 12.969 & 8 & 3 \\
\hline Ardilla (squirrel) & 4.28 & 2.56 & 5.24 & 6.84 & 5.977 & 21 & 3 \\
\hline Avestruz (ostrich) & 3.68 & 1.92 & 6.72 & 6.76 & 7.158 & 4 & 3 \\
\hline Avispa (wasp) & 3.60 & 3.64 & 4.48 & 6.88 & 4.123 & 1 & 3 \\
\hline Ballena (whale) & 5.00 & 3.00 & 5.08 & 6.84 & 11.232 & 4 & 3 \\
\hline Buey (ox) & 4.40 & 2.04 & 5.56 & 6.32 & 3.278 & 6 & 1 \\
\hline Búho (owl) & 3.92 & 2.64 & 5.40 & 6.80 & 6.917 & 12 & 2 \\
\hline Buitre (vulture) & 3.60 & 2.92 & 6.24 & 6.64 & 8.827 & 2 & 2 \\
\hline Burro (donkey) & 5.56 & 4.28 & 3.72 & 6.92 & 23.995 & 17 & 2 \\
\hline Caballo (horse) & 6.48 & 4.20 & 3.64 & 7.00 & 34.723 & 93 & 3 \\
\hline Cabra (goat) & 5.32 & 3.64 & 3.88 & 6.96 & 12.242 & 10 & 2 \\
\hline Calamar (squid) & 2.48 & 3.40 & 5.64 & 6.24 & 6.510 & 1 & 3 \\
\hline Camello (camel) & 4.20 & 2.84 & 5.68 & 6.80 & 2.916 & 4 & 3 \\
\hline Canario (canary) & 4.24 & 3.40 & 4.96 & 6.68 & 15.616 & 7 & 3 \\
\hline Cangrejo (crab) & 3.28 & 2.88 & 4.96 & 6.56 & 2.777 & 2 & 3 \\
\hline Canguro (kangaroo) & 3.92 & 2.48 & 5.96 & 6.84 & 3.801 & 1 & 3 \\
\hline Cebra (zebra) & 4.32 & 2.84 & 6.04 & 6.84 & 6.838 & 3 & 2 \\
\hline Cerdo (pig) & 6.48 & 5.28 & 3.76 & 6.88 & 17.991 & 17 & 2 \\
\hline Ciervo (deer) & 4.92 & 3.08 & 4.98 & 6.84 & 11.601 & 4 & 2 \\
\hline Cigüeña (stork) & 4.60 & 3.84 & 4.82 & 6.64 & 2.644 & 5 & 3 \\
\hline Cocodrilo (crocodile) & 4.20 & 2.36 & 5.16 & 6.76 & 7.753 & 6 & 4 \\
\hline Codorniz (quail) & 3.12 & 2.80 & 7.44 & 5.44 & 5.091 & 2 & 3 \\
\hline Colibrí (hummingbird) & 2.32 & 1.68 & 9.48 & 5.16 & 4.832 & 0 & 3 \\
\hline Colorín (goldfinch) & 1.88 & 1.68 & 7.56 & 4.32 & 4.516 & 1 & 3 \\
\hline Conejo (rabbit) & 6.12 & 4.20 & 3.54 & 6.84 & 30.302 & 9 & 3 \\
\hline Cordero (lamb) & 5.76 & 4.28 & 3.84 & 6.80 & 7.306 & 14 & 3 \\
\hline Cucaracha (cockroach) & 2.80 & 3.20 & 4.56 & 6.84 & 3.545 & 2 & 4 \\
\hline Cuervo (crow) & 3.56 & 2.76 & 4.88 & 6.64 & 10.398 & 4 & 2 \\
\hline Culebra (snake) & 3.84 & 3.24 & 4.68 & 6.84 & 7.098 & 1 & 3 \\
\hline Delfín (dolphin) & 5.44 & 3.20 & 5.40 & 6.88 & 8.164 & 2 & 2 \\
\hline Elefante (elephant) & 6.32 & 3.36 & 4.36 & 6.92 & 21.565 & 9 & 4 \\
\hline Foca (seal) & 4.84 & 3.52 & 5.00 & 6.88 & 3.533 & 1 & 2 \\
\hline Gallina (hen) & 5.88 & 4.88 & 3.36 & 6.88 & 25.464 & 19 & 3 \\
\hline Gallo (rooster) & 5.56 & 4.48 & 3.52 & 6.92 & 11.802 & 16 & 2 \\
\hline Gamba (prawn) & 2.56 & 4.52 & 5.72 & 6.76 & 3.811 & 1 & 2 \\
\hline Gamo (fallow deer) & 3.08 & 1.64 & 8.04 & 5.36 & 2.892 & 1 & 2 \\
\hline Gato (cat) & 6.72 & 5.48 & 3.00 & 6.84 & 76.056 & 70 & 2 \\
\hline Gaviota (seagull) & 4.32 & 2.76 & 5.08 & 6.72 & 5.783 & 6 & 3 \\
\hline Golondrina (swallow) & 4.32 & 3.28 & 5.04 & 6.36 & 6.082 & 6 & 4 \\
\hline Gorila (gorilla) & 4.64 & 2.88 & 5.60 & 6.56 & 5.408 & 4 & 2 \\
\hline Gorrino (pig) & 3.64 & 3.00 & 5.32 & 6.40 & 6.804 & 1 & 3 \\
\hline Gorrión (sparrow) & 3.92 & 3.32 & 5.00 & 6.28 & 11.579 & 1 & 2 \\
\hline
\end{tabular}


APPENDIX (Continued)

\begin{tabular}{|c|c|c|c|c|c|c|c|}
\hline $\begin{array}{c}\text { Spanish Word } \\
\text { (English Translation) }\end{array}$ & Typ & CFam & AoA & Imag & LA & WFreq & Len \\
\hline Guepardo (cheetah) & 3.32 & 2.64 & 7.08 & 5.68 & 2.746 & 0 & 3 \\
\hline Gusano (worm) & 3.12 & 4.12 & 4.00 & 6.72 & 5.827 & 6 & 3 \\
\hline Halcón (falcon) & 3.76 & 2.64 & 6.68 & 6.40 & 3.029 & 2 & 2 \\
\hline Hamster (hamster) & 3.92 & 3.60 & 7.56 & 6.68 & 8.385 & 1 & 2 \\
\hline Hiena (hyena) & 3.08 & 2.28 & 7.16 & 6.24 & 7.051 & 1 & 2 \\
\hline Hipopótamo & & & & & & & \\
\hline (hippopotamus) & 4.44 & 2.28 & 6.16 & 6.84 & 10.631 & 2 & 5 \\
\hline Hormiga (ant) & 4.28 & 3.68 & 4.64 & 6.96 & 9.531 & 5 & 3 \\
\hline Iguana (iguana) & 3.72 & 1.88 & 9.16 & 5.72 & 3.109 & 7 & 3 \\
\hline Jabalí (wild boar) & 5.28 & 2.32 & 8.32 & 6.68 & 12.549 & 5 & 3 \\
\hline Jirafa (giraffe) & 5.40 & 2.16 & 6.48 & 7.00 & 18.874 & 1 & 3 \\
\hline Koala (koala) & 4.52 & 1.68 & 9.00 & 6.67 & 4.209 & 0 & 3 \\
\hline Lagartija (lizard) & 4.04 & 2.64 & 5.64 & 6.68 & 6.384 & 4 & 4 \\
\hline Lagarto (lizard) & 3.80 & 2.04 & 6.76 & 6.32 & 4.210 & 4 & 3 \\
\hline Langostino (prawn) & 3.16 & 3.56 & 8.36 & 6.72 & 4.634 & 0 & 4 \\
\hline Lechuza (owl) & 4.68 & 2.24 & 7.96 & 6.40 & 3.313 & 1 & 3 \\
\hline León (lion) & 6.80 & 3.12 & 5.28 & 6.92 & 37.723 & 27 & 2 \\
\hline Leopardo (leopard) & 6.28 & 2.24 & 7.16 & 6.28 & 12.136 & 1 & 4 \\
\hline Liebre (hare) & 6.40 & 3.00 & 6.16 & 6.52 & 17.270 & 5 & 2 \\
\hline Lince (lynx) & 5.80 & 2.00 & 8.08 & 6.20 & 3.930 & 2 & 2 \\
\hline Lobo (wolf) & 6.76 & 3.20 & 5.44 & 6.72 & 9.537 & 24 & 2 \\
\hline Loro (parrot) & 5.24 & 2.88 & 6.32 & 6.76 & 7.277 & 7 & 2 \\
\hline Mariposa (butterfly) & 4.20 & 3.44 & 4.84 & 6.96 & 8.377 & 8 & 4 \\
\hline Mono (monkey) & 5.96 & 3.32 & 4.92 & 6.80 & 19.337 & 24 & 2 \\
\hline Mosca (fly) & 3.64 & 5.68 & 4.52 & 6.96 & 10.921 & 16 & 2 \\
\hline Mosquito (mosquito) & 3.40 & 4.80 & 5.12 & 6.56 & 5.761 & 4 & 3 \\
\hline Orangután (orangutan) & 5.52 & 1.88 & 7.36 & 6.13 & 4.282 & 2 & 4 \\
\hline Oso (bear) & 6.68 & 2.88 & 5.32 & 6.88 & 16.204 & 22 & 2 \\
\hline Oveja (sheep) & 6.64 & 3.40 & 4.36 & 6.92 & 20.824 & 5 & 3 \\
\hline Pájaro (bird) & 6.12 & 5.60 & 4.08 & 6.80 & 18.035 & 35 & 3 \\
\hline Paloma (pigeon) & 5.64 & 4.88 & 4.96 & 6.92 & 17.684 & 16 & 3 \\
\hline Pantera (panther) & 5.96 & 1.68 & 7.08 & 6.52 & 17.211 & 13 & 3 \\
\hline Pato (duck) & 6.04 & 3.48 & 4.80 & 6.92 & 9.480 & 5 & 2 \\
\hline Perdiz (partridge) & 5.20 & 1.96 & 6.96 & 6.08 & 12.093 & 3 & 2 \\
\hline Periquito (budgerigar) & 4.92 & 2.16 & 7.12 & 6.12 & 18.087 & 0 & 4 \\
\hline Perro (dog) & 6.92 & 6.04 & 3.88 & 7.00 & 82.771 & 112 & 2 \\
\hline Pez (fish) & 5.52 & 5.00 & 4.20 & 6.92 & 13.734 & 30 & 1 \\
\hline Pollo (chicken) & 5.76 & 4.96 & 4.56 & 6.84 & 12.675 & 14 & 2 \\
\hline Potro (colt) & 5.64 & 2.48 & 7.20 & 6.64 & 3.285 & 4 & 2 \\
\hline Puma (puma) & 5.40 & 1.44 & 7.96 & 5.40 & 3.645 & 0 & 2 \\
\hline Rana (frog) & 4.80 & 2.80 & 5.24 & 6.64 & 6.324 & 5 & 2 \\
\hline Rata (rat) & 5.60 & 3.20 & 5.80 & 6.68 & 14.897 & 17 & 2 \\
\hline Ratón (mouse) & 5.72 & 3.16 & 5.08 & 6.80 & 20.367 & 18 & 2 \\
\hline Rinoceronte (rhinoceros) & 5.20 & 1.60 & 7.92 & 6.60 & 6.872 & 1 & 5 \\
\hline Salmón (salmon) & 4.12 & 2.84 & 8.80 & 5.56 & 5.836 & 3 & 2 \\
\hline Saltamontes (grasshopper) & 3.48 & 2.20 & 6.64 & 6.56 & 4.558 & 2 & 4 \\
\hline Sapo (toad) & 4.12 & 2.28 & 6.40 & 6.44 & 5.616 & 5 & 2 \\
\hline Sardina (sardine) & 3.92 & 3.36 & 6.32 & 6.12 & 3.826 & 3 & 3 \\
\hline Serpiente (snake) & 4.76 & 2.88 & 5.60 & 6.84 & 19.985 & 18 & 3 \\
\hline Tiburón (shark) & 5.00 & 2.16 & 6.68 & 6.72 & 10.960 & 1 & 3 \\
\hline Tigre (tiger) & 6.52 & 2.44 & 6.04 & 6.88 & 25.222 & 7 & 2 \\
\hline Toro (bull) & 6.48 & 4.20 & 5.28 & 6.96 & 11.169 & 16 & 2 \\
\hline Tortuga (turtle) & 5.56 & 3.04 & 5.68 & 7.00 & 8.514 & 8 & 3 \\
\hline Trucha (trout) & 4.00 & 3.00 & 7.32 & 5.92 & 7.759 & 3 & 2 \\
\hline Vaca (cow) & 6.72 & 4.32 & 4.52 & 6.96 & 27.320 & 14 & 2 \\
\hline Yegua (mare) & 6.00 & 2.88 & 7.08 & 6.64 & 7.900 & 15 & 2 \\
\hline Zorro (fox) & 6.52 & 3.20 & 5.72 & 6.52 & 10.864 & 4 & 2 \\
\hline \multicolumn{8}{|c|}{ Body Parts } \\
\hline Abdomen (abdomen) & 4.92 & 3.32 & 8.68 & 5.08 & 4.195 & 5 & 3 \\
\hline Ano (anus) & 3.36 & 3.00 & 8.36 & 5.08 & 2.672 & 2 & 2 \\
\hline
\end{tabular}


APPENDIX (Continued)

\begin{tabular}{|c|c|c|c|c|c|c|c|}
\hline $\begin{array}{c}\text { Spanish Word } \\
\text { (English Translation) }\end{array}$ & Typ & CFam & AoA & Imag & LA & WFreq & Len \\
\hline Antebrazo (forearm) & 4.12 & 2.92 & 8.20 & 6.08 & 11.107 & 6 & 4 \\
\hline Arteria (artery) & 2.88 & 2.52 & 8.88 & 4.68 & 1.423 & 1 & 3 \\
\hline Articulaciones (joints) & 3.40 & 3.92 & 8.72 & 4.60 & 1.611 & 3 & 5 \\
\hline Axila (armpit) & 4.24 & 4.48 & 7.84 & 6.32 & 2.444 & 2 & 3 \\
\hline Barba (beard) & 3.52 & 4.80 & 4.04 & 6.88 & 1.045 & 36 & 2 \\
\hline Barbilla (chin) & 5.04 & 4.84 & 4.36 & 6.88 & 1.639 & 16 & 3 \\
\hline Biceps (biceps) & 4.48 & 4.00 & 8.80 & 5.76 & 2.067 & 0 & 2 \\
\hline Bigote (moustache) & 3.72 & 4.68 & 3.88 & 6.92 & 1.106 & 34 & 3 \\
\hline Boca (mouth) & 6.84 & 6.36 & 3.02 & 6.92 & 39.497 & 200 & 2 \\
\hline Brazo (arm) & 7.00 & 6.32 & 2.98 & 6.92 & 71.694 & 96 & 2 \\
\hline Cabello (hair) & 5.40 & 5.60 & 4.72 & 6.84 & 1.078 & 44 & 3 \\
\hline Cabeza (head) & 7.00 & 6.28 & 2.98 & 6.80 & 71.287 & 418 & 3 \\
\hline Cadera (hip) & 5.76 & 4.88 & 5.84 & 6.72 & 12.007 & 7 & 3 \\
\hline Cara (face) & 6.70 & 6.52 & 3.14 & 6.76 & 8.897 & 315 & 2 \\
\hline Ceja (eyebrow) & 4.20 & 5.72 & 3.92 & 6.92 & 12.159 & 8 & 2 \\
\hline Cerebelo (cerebellum) & 2.44 & 1.68 & 9.79 & 4.16 & 1.001 & 1 & 4 \\
\hline Cerebro (brain) & 4.28 & 4.36 & 7.44 & 5.28 & 7.956 & 87 & 3 \\
\hline Cintura (waist) & 5.20 & 5.16 & 5.72 & 6.00 & 5.811 & 34 & 3 \\
\hline Clavícula (collarbone) & 4.48 & 3.24 & 8.68 & 5.36 & 1.143 & 1 & 4 \\
\hline Codo (elbow) & 5.28 & 4.92 & 5.48 & 6.28 & 29.918 & 17 & 2 \\
\hline Colon (colon) & 2.48 & 2.04 & 10.44 & 3.68 & 1.058 & 0 & 2 \\
\hline Corazón (heart) & 5.36 & 5.44 & 5.44 & 6.40 & 17.079 & 190 & 3 \\
\hline Costilla (rib) & 4.92 & 4.20 & 6.48 & 6.44 & 1.918 & 2 & 3 \\
\hline Cubito (fibula) & 2.32 & 2.24 & 9.60 & 4.64 & 1.253 & 1 & 3 \\
\hline Cuello (neck) & 5.88 & 5.60 & 4.08 & 6.64 & 31.656 & 99 & 2 \\
\hline Culo (bottom) & 6.04 & 5.76 & 3.12 & 6.76 & 13.216 & 37 & 2 \\
\hline Dedo (finger) & 6.36 & 6.04 & 3.04 & 6.92 & 53.948 & 66 & 2 \\
\hline Diente (tooth) & 5.56 & 6.40 & 3.78 & 6.88 & 19.575 & 8 & 2 \\
\hline Esófago (esophagus) & 2.84 & 2.72 & 8.84 & 4.40 & 3.474 & 2 & 4 \\
\hline Espalda (back) & 6.48 & 5.92 & 4.40 & 6.76 & 19.081 & 92 & 3 \\
\hline Esqueleto (skeleton) & 4.00 & 3.32 & 6.56 & 6.56 & 0.982 & 14 & 4 \\
\hline \multicolumn{8}{|l|}{ Esternocleidomastoideo } \\
\hline (a neck muscle) & 2.44 & 2.04 & 10.95 & 2.60 & 4.144 & 0 & 9 \\
\hline Estómago (stomach) & 4.84 & 5.32 & 6.56 & 5.28 & 12.166 & 43 & 4 \\
\hline Extremidades (extremities) & 4.76 & 4.04 & 7.04 & 6.28 & 3.855 & 5 & 5 \\
\hline Falange (phalange) & 2.64 & 2.52 & 9.13 & 4.76 & 4.685 & 4 & 3 \\
\hline Faringe (pharynx) & 3.04 & 2.60 & 9.12 & 4.24 & 2.642 & 1 & 3 \\
\hline Fémur (femur) & 3.36 & 2.48 & 9.24 & 5.08 & 2.564 & 0 & 2 \\
\hline Frente (forehead) & 5.56 & 4.96 & 4.88 & 6.48 & 5.747 & 375 & 2 \\
\hline Gemelo (calf muscle) & 4.08 & 4.76 & 8.68 & 6.12 & 3.222 & 3 & 3 \\
\hline Glúteo (gluteus) & 4.24 & 3.88 & 8.92 & 6.48 & 4.044 & 1 & 3 \\
\hline Hígado (liver) & 4.16 & 4.00 & 7.92 & 5.56 & 7.299 & 12 & 3 \\
\hline Hombro (shoulder) & 5.72 & 5.32 & 4.68 & 6.80 & 29.002 & 45 & 2 \\
\hline Hueso (bone) & 4.96 & 5.08 & 4.40 & 6.68 & 5.329 & 22 & 2 \\
\hline Húmero (humerus) & 2.96 & 2.40 & 8.60 & 4.60 & 1.576 & 0 & 3 \\
\hline Incisivo (incisor) & 2.80 & 2.44 & 8.60 & 5.00 & 93.5 & 2 & 4 \\
\hline Ingle (groin) & 4.00 & 3.48 & 8.60 & 5.56 & 110 & 3 & 2 \\
\hline Intestino (intestine) & 3.64 & 3.04 & 8.08 & 4.92 & 6.1 & 3 & 3 \\
\hline Labio (lip) & 6.08 & 6.04 & 4.30 & 6.92 & 8.3 & 12 & 2 \\
\hline Laringe (larynx) & 3.36 & 2.00 & 9.84 & 4.36 & 3.3 & 2 & 3 \\
\hline Lengua (tongue) & 5.44 & 5.80 & 4.72 & 6.88 & 13.5 & $17 \overline{7}$ & 2 \\
\hline Mano (hand) & 6.92 & 6.56 & 3.96 & 6.96 & 63.0 & 550 & 2 \\
\hline Mejilla (cheek) & 5.52 & 4.96 & 6.56 & 6.84 & 0.9 & 27 & 3 \\
\hline Moflete (chubby cheek) & 5.16 & 4.48 & 5.80 & 6.80 & 1.0 & 4 & 3 \\
\hline Muela (molar) & 3.92 & 4.12 & 6.20 & 6.96 & 2.4 & 2 & 3 \\
\hline Muñeca (wrist) & 5.64 & 4.20 & 5.56 & 6.88 & 21.2 & 16 & 3 \\
\hline Músculo (muscle) & 4.60 & 4.04 & 7.44 & 5.68 & 6.5 & 9 & 3 \\
\hline Muslo (thigh) & 5.40 & 3.84 & 7.36 & 6.76 & 6.7 & 11 & 2 \\
\hline Nariz (nose) & 6.48 & 5.48 & 4.16 & 6.96 & 50.4 & 71 & 2 \\
\hline Nuca (nape) & 5.12 & 3.48 & 6.80 & 6.36 & 2.1 & 23 & 2 \\
\hline Oído (ear) & 5.48 & 5.24 & 5.16 & 5.80 & 2.9 & 107 & 3 \\
\hline
\end{tabular}


APPENDIX (Continued)

\begin{tabular}{|c|c|c|c|c|c|c|c|}
\hline $\begin{array}{c}\text { Spanish Word } \\
\text { (English Translation) }\end{array}$ & Typ & CFam & AoA & Imag & LA & WFreq & Len \\
\hline Ojo (eye) & 6.64 & 6.24 & 4.08 & 6.76 & 60.1 & 96 & 2 \\
\hline Ombligo (belly button) & 4.68 & 4.12 & 5.40 & 6.96 & 8.3 & 8 & 3 \\
\hline \multicolumn{8}{|l|}{ Omóplato } \\
\hline (shoulder blade) & 3.48 & 2.16 & 9.24 & 5.52 & 2.6 & 1 & 4 \\
\hline Oreja (ear) & 6.56 & 4.92 & 4.32 & 6.84 & 48.1 & 35 & 3 \\
\hline Ovario (ovary) & 3.32 & 3.16 & 10.48 & 4.44 & 1.6 & 1 & 3 \\
\hline Pancreas (pancreas) & 3.12 & 1.72 & 10.80 & 4.04 & 4.7 & 0 & 3 \\
\hline Pantorrilla (calf) & 4.68 & 2.40 & 8.92 & 5.92 & 1.4 & 2 & 4 \\
\hline Párpado (eyelid) & 4.16 & 3.36 & 7.92 & 6.68 & 2.4 & 3 & 3 \\
\hline Pecho (chest) & 6.12 & 4.08 & 6.76 & 6.56 & 21.7 & 94 & 2 \\
\hline Pechos (breasts) & 5.96 & 4.96 & 7.52 & 6.72 & 2.2 & 34 & 2 \\
\hline Pelo (hair) & 5.36 & 6.24 & 4.40 & 6.92 & 27.9 & 153 & 2 \\
\hline Pelvis (pelvis) & 4.00 & 2.32 & 10.48 & 5.64 & 1.1 & 1 & 2 \\
\hline Pene (penis) & 5.36 & 3.52 & 9.68 & 6.72 & 16.1 & 7 & 2 \\
\hline Peroné (fibula) & 3.40 & 1.96 & 9.76 & 5.12 & 2.2 & 0 & 3 \\
\hline Pestaña (eyelash) & 3.92 & 4.20 & 6.44 & 6.88 & 8.7 & 1 & 3 \\
\hline Pie (foot) & 6.60 & 5.96 & 4.08 & 7.00 & 61.8 & 193 & 1 \\
\hline Piel (skin) & 4.52 & 5.40 & 5.44 & 6.20 & 1.0 & 163 & 1 \\
\hline Pierna (leg) & 6.68 & 5.92 & 4.64 & 6.60 & 58.1 & 39 & 2 \\
\hline Pulmón (lung) & 4.36 & 4.08 & 8.28 & 5.84 & 13.2 & 6 & 2 \\
\hline Radio (radius) & 2.88 & 2.44 & 8.52 & 5.20 & 1.9 & 75 & 2 \\
\hline Riñón (kidney) & 4.24 & 2.80 & 9.04 & 5.52 & 11.7 & 5 & 2 \\
\hline Rodilla (knee) & 5.92 & 4.04 & 6.00 & 6.56 & 31.7 & 11 & 3 \\
\hline Rótula (kneecap) & 3.48 & 2.36 & 10.28 & 5.80 & 1.5 & 0 & 3 \\
\hline Talón (heel) & 4.60 & 2.36 & 8.24 & 6.52 & 2.6 & 3 & 2 \\
\hline Tendón (tendon) & 3.36 & 2.36 & 9.84 & 5.04 & 2.2 & 1 & 2 \\
\hline Testículo (testicle) & 4.28 & 2.64 & 9.68 & 6.72 & 4.1 & 4 & 4 \\
\hline Tibia (tibia) & 3.56 & 1.92 & 9.24 & 5.40 & 2.8 & 14 & 2 \\
\hline Tobillo (ankle) & 5.36 & 3.24 & 6.84 & 6.48 & 20.9 & 4 & 3 \\
\hline Tórax (thorax) & 4.52 & 2.00 & 9.60 & 6.00 & 3.2 & 6 & 2 \\
\hline Trasero (bottom) & 5.60 & 3.56 & 7.12 & 6.88 & 1.3 & 19 & 3 \\
\hline Triceps (triceps) & 3.36 & 2.24 & 10.95 & 5.56 & 1.4 & 0 & 2 \\
\hline Tripa (belly) & 5.24 & 4.52 & 6.32 & 6.72 & 3.4 & 9 & 2 \\
\hline Tronco (trunk) & 5.24 & 2.80 & 8.14 & 6.36 & 15.3 & 18 & 2 \\
\hline Uña (nail) & 4.80 & 5.28 & 6.18 & 6.84 & 27.6 & 8 & 2 \\
\hline Vagina (vagina) & 4.16 & 2.96 & 9.95 & 6.28 & 3.8 & 4 & 3 \\
\hline Vejiga (bladder) & 3.72 & 2.92 & 9.73 & 5.56 & 1.0 & 1 & 3 \\
\hline Vena (vein) & 3.48 & 3.24 & 8.73 & 5.88 & 2.4 & 5 & 2 \\
\hline \multicolumn{8}{|c|}{ Furniture } \\
\hline Alacena (cupboard) & 3.36 & 1.92 & 8.23 & 5.28 & 2.394 & 4 & 4 \\
\hline Alfombra (rug) & 3.40 & 5.64 & 4.58 & 6.60 & 9.652 & 22 & 3 \\
\hline Aparador (sideboard) & 3.68 & 2.76 & 8.00 & 5.44 & 7.180 & 7 & 4 \\
\hline Arca (chest) & 3.40 & 2.28 & 6.88 & 6.08 & 2.423 & 11 & 2 \\
\hline Arcón (chest) & 3.40 & 2.80 & 7.04 & 6.32 & 1.355 & 3 & 2 \\
\hline Armario (wardrobe) & 7.00 & 6.36 & 4.00 & 6.72 & 63.039 & 38 & 3 \\
\hline Balancín (seesaw) & 2.88 & 2.52 & 6.48 & 5.92 & 2.785 & 1 & 3 \\
\hline Banco (bench) & 4.92 & 5.52 & 4.04 & 6.72 & 0.870 & 52 & 2 \\
\hline Banqueta (stool) & 4.92 & 4.08 & 5.38 & 6.44 & 5.731 & 3 & 3 \\
\hline Bañera (bath) & 3.60 & 5.96 & 4.08 & 6.88 & 4.623 & 8 & 3 \\
\hline Barandilla (railing) & 2.64 & 4.72 & 4.64 & 6.72 & 0.910 & 5 & 4 \\
\hline Barra (bar) & 1.88 & 4.52 & 5.32 & 6.16 & 0.719 & 45 & 2 \\
\hline Baúl (trunk) & 5.04 & 3.60 & 4.80 & 6.64 & 9.128 & 8 & 2 \\
\hline Bidé (bidet) & 3.44 & 4.72 & 5.68 & 6.40 & 2.313 & 1 & 2 \\
\hline Buró (bureau) & 3.16 & 1.16 & 11.00 & 4.16 & 1.574 & 1 & 2 \\
\hline Butaca (armchair) & 5.60 & 4.32 & 5.96 & 6.44 & 4.788 & 18 & 3 \\
\hline Cajón (drawer) & 5.76 & 5.84 & 3.88 & 6.84 & 5.344 & 18 & 2 \\
\hline \multicolumn{8}{|l|}{ Cajonera } \\
\hline (chest of drawers) & 5.12 & 4.12 & 6.13 & 6.52 & 3.218 & 2 & 4 \\
\hline Cama (bed) & 6.92 & 6.76 & 3.08 & 6.84 & 67.877 & 199 & 2 \\
\hline Cocina (stove) & 4.36 & 6.24 & 3.16 & 6.72 & 4.895 & 112 & 3 \\
\hline Cómoda (sideboard) & 5.68 & 4.08 & 6.80 & 6.32 & 13.009 & 14 & 3 \\
\hline
\end{tabular}


APPENDIX (Continued)

\begin{tabular}{|c|c|c|c|c|c|c|c|}
\hline $\begin{array}{c}\text { Spanish Word } \\
\text { (English Translation) }\end{array}$ & Typ & CFam & AoA & Imag & LA & WFreq & Len \\
\hline \multicolumn{8}{|l|}{ Comodín } \\
\hline (chest of drawers) & 3.52 & 2.68 & 7.30 & 4.96 & 3.633 & 1 & 3 \\
\hline Consola (console table) & 3.44 & 3.28 & 10.92 & 4.92 & 1.424 & 7 & 3 \\
\hline Coqueta (dressing table) & 3.36 & 2.88 & 10.56 & 4.20 & 2.281 & 4 & 3 \\
\hline Cortina (curtain) & 3.68 & 5.36 & 4.00 & 6.68 & 0.745 & 20 & 3 \\
\hline Cuadro (picture) & 3.80 & 5.24 & 3.76 & 6.56 & 16.830 & 61 & 2 \\
\hline Descalzadora (shoe horn) & 1.84 & 1.84 & 8.86 & 4.68 & 0.850 & 1 & 5 \\
\hline Diván (divan) & 3.92 & 1.92 & 10.52 & 5.76 & 1.468 & 10 & 2 \\
\hline Ducha (shower) & 3.24 & 6.52 & 5.56 & 6.76 & 1.870 & 21 & 2 \\
\hline Encimera (worktop) & 4.48 & 5.12 & 7.79 & 6.40 & 9.094 & 0 & 4 \\
\hline Entradita (entrance hall) & 2.68 & 2.92 & 9.19 & 5.44 & 2.156 & 0 & 4 \\
\hline Escabel (footstool) & 2.00 & 1.16 & 11.18 & 2.88 & 0.641 & 2 & 3 \\
\hline Escritor (desk) & 5.88 & 5.80 & 7.46 & 6.76 & 28.341 & 14 & 4 \\
\hline Escurreplatos (plate rack) & 3.24 & 4.52 & 7.88 & 6.68 & 0.842 & 0 & 5 \\
\hline Espejo (mirror) & 4.56 & 6.32 & 3.76 & 6.92 & 13.704 & 74 & 3 \\
\hline Estante (shelf) & 5.52 & 5.04 & 5.46 & 6.80 & 0.699 & 4 & 3 \\
\hline Estantería (shelves) & 6.28 & 6.24 & 5.28 & 6.88 & 44.794 & 5 & 5 \\
\hline Flexo (reading lamp) & 3.52 & 6.12 & 8.20 & 6.32 & 1.925 & 2 & 2 \\
\hline Fogón (ring) & 2.64 & 3.16 & 7.71 & 6.08 & 0.679 & 4 & 2 \\
\hline Fregadero (sink) & 4.08 & 4.76 & 5.52 & 6.80 & 1.464 & 6 & 4 \\
\hline Fregador (sink) & 2.08 & 3.00 & 7.08 & 4.96 & 1.343 & 0 & 3 \\
\hline Frigorífico (fridge) & 4.52 & 5.68 & 5.08 & 6.84 & 6.182 & 3 & 5 \\
\hline Guardarropa (wardrobe) & 4.96 & 3.08 & 8.63 & 6.52 & 1.304 & 3 & 4 \\
\hline Hamaca (hammock) & 3.44 & 2.60 & 8.00 & 6.60 & 7.290 & 5 & 3 \\
\hline Horno (oven) & 3.36 & 4.76 & 5.08 & 6.80 & 5.384 & 6 & 2 \\
\hline Jarrón (vase) & 2.88 & 4.44 & 4.44 & 6.76 & 0.857 & 5 & 2 \\
\hline Lámpara (lamp) & 3.80 & 5.64 & 3.96 & 6.76 & 24.219 & 22 & 3 \\
\hline Lavabo (washbasin) & 3.80 & 6.16 & 4.27 & 6.68 & 6.246 & 18 & 3 \\
\hline \multicolumn{8}{|l|}{ Lavadora } \\
\hline (washing machine) & 2.80 & 5.16 & 7.16 & 6.64 & 6.740 & 2 & 4 \\
\hline Lavaplatos (dishwasher) & 2.88 & 4.44 & 8.33 & 6.60 & 0.935 & 1 & 4 \\
\hline Lavavajillas (dishwasher) & 2.56 & 4.00 & 9.16 & 6.84 & 3.946 & 1 & 5 \\
\hline Librería (bookcase) & 5.40 & 4.76 & 7.04 & 6.68 & 3.829 & 9 & 4 \\
\hline Litera (bunk bed) & 5.08 & 2.36 & 8.28 & 6.92 & 4.466 & 1 & 3 \\
\hline Mecedora (rocking chair) & 5.16 & 2.24 & 8.20 & 6.76 & 5.343 & 4 & 4 \\
\hline Mesa (table) & 6.92 & 6.76 & 4.20 & 7.00 & 78.615 & 228 & 2 \\
\hline Mesita (bedside table) & 6.36 & 5.48 & 5.04 & 6.84 & 42.025 & 2 & 3 \\
\hline Microondas (microwaves) & 2.64 & 5.32 & 11.42 & 7.00 & 8.237 & 3 & 4 \\
\hline Minicadena (mini hi-fi) & 2.28 & 5.44 & 10.88 & 6.96 & 1.890 & 0 & 5 \\
\hline Mural (mural) & 2.00 & 2.12 & 9.00 & 5.04 & 0.641 & 3 & 2 \\
\hline Nevera (fridge) & 2.72 & 5.96 & 6.52 & 6.96 & 6.282 & 12 & 3 \\
\hline Nido (truckle bed) & 3.28 & 1.88 & 6.92 & 6.00 & 0.900 & 13 & 2 \\
\hline Ordenador (computer) & 2.24 & 6.44 & 10.75 & 6.96 & 4.671 & 67 & 4 \\
\hline Papelera (waste basket) & 2.84 & 6.00 & 6.24 & 7.00 & 2.398 & 4 & 4 \\
\hline \multicolumn{8}{|l|}{ Paragüero } \\
\hline (umbrella stand) & 3.04 & 3.28 & 7.60 & 6.76 & 1.694 & 1 & 4 \\
\hline Percha (hanger) & 2.76 & 5.28 & 6.40 & 6.96 & 4.559 & 7 & 2 \\
\hline Perchero (clothes stand) & 3.84 & 3.88 & 7.12 & 6.68 & 5.286 & 4 & 3 \\
\hline Piano (piano) & 2.20 & 3.28 & 6.04 & 7.00 & 1.858 & 31 & 3 \\
\hline Portalibros (book shelf) & 2.84 & 2.28 & 11.15 & 5.20 & 0.641 & 0 & 4 \\
\hline Posaplatos (place mat) & 2.04 & 2.36 & 11.64 & 5.36 & 0.679 & 0 & 4 \\
\hline Puerta (door) & 3.48 & 6.56 & 4.24 & 7.00 & 4.876 & 301 & 2 \\
\hline Puf (pouffe) & 3.00 & 2.48 & 10.86 & 5.08 & 3.812 & 0 & 1 \\
\hline Pupitre (school desk) & 5.44 & 3.68 & 6.00 & 6.76 & 1.752 & 9 & 3 \\
\hline Radio (radio) & 2.24 & 5.76 & 5.68 & 6.96 & 1.787 & 75 & 2 \\
\hline Recibidor (hall) & 3.88 & 3.16 & 8.72 & 5.96 & 2.872 & 7 & 4 \\
\hline Reloj (clock) & 2.52 & 6.24 & 4.92 & 7.00 & 2.519 & 43 & 2 \\
\hline Repisa (shelf) & 3.60 & 4.04 & 7.84 & 6.64 & 1.000 & 3 & 3 \\
\hline Retrete (toilet) & 1.80 & 3.36 & 8.64 & 7.00 & 1.261 & 14 & 3 \\
\hline Revistero (magazine rack) & 3.44 & 2.80 & 10.25 & 6.20 & 0.914 & 0 & 4 \\
\hline Rinconera (cornerboard) & 4.84 & 2.44 & 11.58 & 5.76 & 1.990 & 0 & 4 \\
\hline Ropero (wardrobe) & 6.08 & 3.32 & 9.84 & 6.48 & 2.893 & 6 & 3 \\
\hline
\end{tabular}


APPENDIX (Continued)

\begin{tabular}{|c|c|c|c|c|c|c|c|}
\hline $\begin{array}{c}\text { Spanish Word } \\
\text { (English Translation) }\end{array}$ & Typ & CFam & AoA & Imag & LA & WFreq & Len \\
\hline Secreter (writing desk) & 4.44 & 1.36 & 16.89 & 4.12 & 1.215 & 0 & 3 \\
\hline Silla (chair) & 6.80 & 6.72 & 4.64 & 7.00 & 78.805 & 58 & 2 \\
\hline Sillón (armchair) & 6.76 & 5.84 & 5.00 & 7.00 & 61.626 & 31 & 2 \\
\hline \multicolumn{8}{|l|}{ Sinfonier } \\
\hline (chest of drawers) & 4.20 & 1.64 & 13.69 & 3.24 & 3.479 & 0 & 3 \\
\hline Sofá (sofa) & 6.80 & 6.36 & 6.24 & 7.00 & 56.795 & 4 & 2 \\
\hline Taburete (stool) & 5.72 & 4.48 & 6.08 & 6.96 & 18.160 & 2 & 4 \\
\hline \multicolumn{8}{|l|}{ Taquillón (traditional } \\
\hline Spanish table) & 4.84 & 2.32 & 9.95 & 5.52 & 2.278 & 0 & 3 \\
\hline Televisión (television) & 4.00 & 6.24 & 4.35 & 6.80 & 14.852 & 85 & 4 \\
\hline Televisor (television) & 2.88 & 5.76 & 6.52 & 6.96 & 2.819 & 25 & 4 \\
\hline Tiestos (flowerpots) & 1.56 & 3.48 & 5.80 & 6.92 & 0.641 & 3 & 2 \\
\hline Tocador (dressing table) & 5.24 & 1.80 & 9.16 & 6.32 & 5.394 & 5 & 3 \\
\hline Tresillo (sofa) & 6.04 & 2.88 & 9.96 & 5.96 & 8.622 & 4 & 3 \\
\hline Tumbona (deck chair) & 4.32 & 2.04 & 9.72 & 6.52 & 1.454 & 2 & 3 \\
\hline Váter (toilet) & 1.68 & 5.80 & 5.36 & 6.56 & 2.025 & 1 & 2 \\
\hline Ventana (window) & 2.20 & 6.56 & 4.36 & 6.96 & 3.336 & 120 & 3 \\
\hline Video (video) & 1.84 & 5.52 & 10.60 & 7.00 & 1.808 & 30 & 3 \\
\hline \multicolumn{8}{|l|}{ Vidriera (stained } \\
\hline glass window) & 1.72 & 2.36 & 10.36 & 6.56 & 1.463 & 5 & 3 \\
\hline Vitrina (glass cabinet) & 4.12 & 2.64 & 10.60 & 6.44 & 2.906 & 6 & 3 \\
\hline WC (toilet) & 1.88 & 3.64 & 9.69 & 6.68 & 2.658 & 0 & 2 \\
\hline Zapatero (shoe rack) & 4.56 & 2.60 & 7.24 & 6.20 & 8.043 & 0 & 4 \\
\hline \multicolumn{8}{|c|}{ Clothing } \\
\hline Abrigo (coat) & 6.00 & 5.56 & 3.80 & 6.76 & 33.065 & 78 & 3 \\
\hline Albornoz (bathrobe) & 3.00 & 4.20 & 8.32 & 6.84 & 0.796 & 2 & 3 \\
\hline Americana (jacket) & 4.16 & 1.80 & 9.40 & 6.52 & 4.846 & 27 & 5 \\
\hline Anillo (ring) & 2.20 & 4.36 & 4.44 & 6.96 & 0.938 & 20 & 3 \\
\hline Anorak (anorak) & 3.88 & 3.00 & 8.40 & 6.32 & 6.798 & 2 & 3 \\
\hline Bañador (swimsuit) & 3.76 & 2.20 & 4.32 & 6.92 & 6.461 & 5 & 3 \\
\hline Bata (robe) & 3.40 & 3.04 & 4.60 & 6.96 & 1.399 & 25 & 2 \\
\hline \multicolumn{8}{|l|}{ Bermudas } \\
\hline (bermuda shorts) & 3.52 & 1.84 & 8.28 & 6.80 & 2.927 & 0 & 3 \\
\hline Biquini (bikini) & 3.44 & 2.76 & 8.12 & 6.92 & 3.990 & 1 & 3 \\
\hline Blusa (blouse) & 5.76 & 2.76 & 6.56 & 6.80 & 9.950 & 15 & 2 \\
\hline Body (body stocking) & 2.76 & 2.04 & 10.42 & 5.88 & 1.137 & 0 & 2 \\
\hline Boina (beret) & 2.16 & 1.52 & 6.04 & 6.72 & 1.246 & 7 & 2 \\
\hline Bolsa (bag) & 1.40 & 5.44 & 4.28 & 6.52 & 0.807 & 34 & 2 \\
\hline Bolso (bag) & 2.56 & 5.76 & 4.72 & 6.92 & 2.066 & 19 & 2 \\
\hline Bota (boot) & 3.44 & 5.80 & 4.36 & 6.92 & 17.199 & 26 & 2 \\
\hline Botines (ankle boot) & 2.48 & 2.96 & 7.72 & 6.80 & 2.672 & 4 & 3 \\
\hline Botón (button) & 1.60 & 3.60 & 4.40 & 6.92 & 1.662 & 25 & 2 \\
\hline Bragas (panties) & 4.44 & 5.28 & 3.64 & 6.88 & 45.394 & 9 & 2 \\
\hline Bufanda (scarf) & 4.16 & 5.16 & 3.88 & 6.84 & 31.043 & 6 & 3 \\
\hline Calcetines (socks) & 4.92 & 5.80 & 3.44 & 6.92 & 58.667 & 12 & 3 \\
\hline Calzoncillos (underpants) & 4.64 & 4.04 & 3.84 & 6.88 & 46.910 & 5 & 4 \\
\hline Calzones (underpants) & 3.88 & 2.40 & 6.24 & 6.52 & 1.592 & 2 & 3 \\
\hline Camisa (shirt) & 6.48 & 4.80 & 4.28 & 6.84 & 62.484 & 61 & 3 \\
\hline Camiseta (T-shirt) & 6.64 & 6.16 & 3.64 & 7.00 & 64.236 & 12 & 4 \\
\hline Camisón (night dress) & 3.80 & 2.12 & 4.68 & 6.76 & 7.354 & 8 & 3 \\
\hline Capa (cloak) & 2.60 & 1.52 & 6.16 & 6.44 & 0.928 & 43 & 2 \\
\hline Cazadora (jacket) & 6.00 & 6.16 & 6.84 & 6.80 & 20.456 & 16 & 4 \\
\hline Chal (shawl) & 3.13 & 2.36 & 9.76 & 6.40 & 2.255 & 2 & 1 \\
\hline Chaleco (vest) & 4.20 & 2.68 & 6.72 & 6.72 & 13.603 & 18 & 3 \\
\hline Chancla (flip-flop) & 2.36 & 3.08 & 7.24 & 6.68 & 6.211 & 1 & 2 \\
\hline Chandal (track suit) & 5.64 & 5.16 & 6.60 & 6.68 & 30.959 & 0 & 2 \\
\hline Chaqueta (jacket) & 6.16 & 4.92 & 4.64 & 6.76 & 37.792 & 33 & 3 \\
\hline Chaquetón (short coat) & 4.88 & 2.88 & 6.52 & 6.56 & 3.743 & 2 & 3 \\
\hline Chubasquero (raincoat) & 4.36 & 3.00 & 7.76 & 6.64 & 6.823 & 1 & 4 \\
\hline Chupa (leather jacket) & 3.92 & 3.00 & 12.76 & 6.48 & 1.920 & 2 & 2 \\
\hline Cinturón (belt) & 3.16 & 4.64 & 5.60 & 6.84 & 7.745 & 23 & 3 \\
\hline Collares (necklaces) & 1.72 & 4.12 & 4.52 & 6.80 & 1.265 & 6 & 3 \\
\hline
\end{tabular}


APPENDIX (Continued)

\begin{tabular}{|c|c|c|c|c|c|c|c|}
\hline $\begin{array}{c}\text { Spanish Word } \\
\text { (English Translation) }\end{array}$ & Typ & CFam & AoA & Imag & LA & WFreq & Len \\
\hline Corbata (tie) & 3.60 & 2.60 & 5.36 & 6.84 & 7.711 & 26 & 3 \\
\hline Correa (belt) & 1.96 & 1.76 & 6.20 & 6.52 & 8.000 & 4 & 3 \\
\hline Corsé (corset) & 2.32 & 1.20 & 10.64 & 6.12 & 0.831 & 2 & 2 \\
\hline Deportivas (trainers) & 3.80 & 5.92 & 8.20 & 6.60 & 4.521 & 1 & 4 \\
\hline Diadema (hairband) & 1.84 & 2.72 & 5.16 & 6.44 & 1.288 & 4 & 3 \\
\hline Ejecutivos (sock) & 2.20 & 2.08 & 13.10 & 5.68 & 0.843 & 7 & 5 \\
\hline Faja (girdle) & 2.60 & 1.36 & 8.00 & 6.04 & 5.029 & 3 & 2 \\
\hline Falda (skirt) & 6.44 & 4.32 & 4.04 & 6.84 & 34.140 & 36 & 2 \\
\hline Frac (tailcoat) & 3.12 & 1.24 & 11.68 & 6.04 & 0.831 & 2 & 1 \\
\hline Fular (foulard scarf) & 3.04 & 1.96 & 11.65 & 6.04 & 3.544 & 2 & 2 \\
\hline Gabardina (raincoat) & 5.00 & 2.52 & 8.28 & 6.64 & 4.663 & 9 & 4 \\
\hline Gorra (cap) & 3.64 & 4.20 & 4.84 & 6.60 & 8.258 & 13 & 2 \\
\hline Gorro (hat) & 3.60 & 4.00 & 4.96 & 6.72 & 19.931 & 7 & 2 \\
\hline Guante (glove) & 3.28 & 4.36 & 5.64 & 6.88 & 24.063 & 16 & 2 \\
\hline Impermeable (raincoat) & 3.68 & 2.92 & 7.61 & 6.44 & 2.088 & 4 & 4 \\
\hline Jeans (jeans) & 5.92 & 3.08 & 10.29 & 6.64 & 1.284 & 0 & 1 \\
\hline Jersey (pullover) & 6.56 & 5.52 & 5.78 & 6.84 & 70.819 & 10 & 2 \\
\hline Leotardo (woolen tights) & 3.16 & 2.76 & 7.83 & 6.48 & 5.250 & 1 & 4 \\
\hline Liga (garter) & 1.76 & 1.16 & 10.21 & 6.56 & 1.823 & 8 & 2 \\
\hline Mallas (leotard) & 3.28 & 1.72 & 10.16 & 6.48 & 4.771 & 2 & 2 \\
\hline Manoplas (mittens) & 2.68 & 2.04 & 7.20 & 6.56 & 3.166 & 0 & 3 \\
\hline Medias (tights) & 3.44 & 3.64 & 7.08 & 6.92 & 25.545 & 49 & 2 \\
\hline Minifalda (miniskirt) & 5.48 & 3.20 & 9.28 & 6.96 & 5.097 & 5 & 4 \\
\hline Mono (dungarees) & 3.76 & 1.92 & 8.83 & 6.56 & 2.196 & 24 & 2 \\
\hline Muñequera (wristband) & 1.28 & 1.80 & 9.60 & 6.64 & 1.647 & 0 & 4 \\
\hline Niqui (T-shirt) & 5.84 & 3.48 & 9.92 & 6.44 & 1.913 & 0 & 2 \\
\hline Orejeras (earflaps) & 1.72 & 1.40 & 9.08 & 6.56 & 3.677 & 3 & 4 \\
\hline Pajarita (bow tie) & 1.64 & 1.44 & 8.92 & 6.80 & 1.787 & 2 & 4 \\
\hline \multicolumn{8}{|l|}{ Palestina/o } \\
\hline (Palestinian scarf) & 1.72 & 2.92 & 13.55 & 5.96 & 1.449 & 2 & 4 \\
\hline Pantaloon (trousers) & 6.72 & 6.68 & 4.44 & 6.76 & 70.674 & 35 & 3 \\
\hline Panty (tights) & 3.36 & 2.56 & 9.52 & 6.20 & 4.560 & 1 & 2 \\
\hline Pañuelo (handkerchief) & 2.44 & 5.08 & 5.76 & 6.80 & 5.489 & 27 & 3 \\
\hline Pareo (beach wrap) & 2.84 & 2.20 & 14.26 & 6.29 & 0.843 & 0 & 3 \\
\hline Peto (dungarees) & 4.68 & 1.96 & 9.48 & 6.60 & 2.732 & 3 & 2 \\
\hline Peúcos (bootees) & 1.92 & 1.04 & 15.60 & 2.00 & 0.963 & 0 & 3 \\
\hline Pichi (pinafore dress) & 4.00 & 1.28 & 8.58 & 4.67 & 1.025 & 1 & 2 \\
\hline Pijama (pyjama) & 5.16 & 5.84 & 4.80 & 6.84 & 10.423 & 9 & 3 \\
\hline Plumas (quilted anorak) & 5.16 & 4.72 & 7.92 & 6.76 & 12.881 & 23 & 2 \\
\hline \multicolumn{8}{|l|}{ Plumífero } \\
\hline (quilted anorak) & 4.52 & 3.84 & 11.88 & 6.24 & 0.894 & 0 & 4 \\
\hline Polar (fleece) & 5.48 & 5.00 & 11.79 & 6.48 & 10.771 & 2 & 2 \\
\hline Polo (polo shirt) & 6.12 & 3.60 & 9.92 & 6.72 & 7.966 & 25 & 2 \\
\hline Poncho (poncho) & 3.76 & 2.20 & 12.33 & 6.64 & 1.769 & 2 & 2 \\
\hline Pulsera (bracelet) & 1.32 & 4.00 & 6.24 & 6.64 & 1.216 & 7 & 3 \\
\hline Rebeca (cardigan) & 5.20 & 2.72 & 10.17 & 5.92 & 1.847 & 1 & 3 \\
\hline Reloj (watch) & 1.48 & 6.48 & 5.12 & 6.96 & 1.842 & 72 & 2 \\
\hline Rodillera (kneeband) & 1.28 & 2.08 & 8.64 & 6.12 & 0.799 & 1 & 4 \\
\hline Rokys (rokys) & 2.00 & 1.00 & 14.14 & 2.68 & 0.883 & 0 & 2 \\
\hline Sandalia (sandal) & 2.92 & 2.48 & 7.48 & 6.44 & 3.759 & 2 & 3 \\
\hline Shorts (shorts) & 4.80 & 1.80 & 12.54 & 5.88 & 1.737 & 0 & 1 \\
\hline Slips (slips) & 4.24 & 3.08 & 11.46 & 6.36 & 1.412 & 0 & 1 \\
\hline Sombrero (hat) & 2.76 & 2.80 & 5.48 & 6.92 & 5.212 & 44 & 3 \\
\hline Sudadera (sweater) & 6.40 & 5.04 & 8.83 & 6.92 & 37.104 & 0 & 4 \\
\hline Suéter (sweater) & 6.56 & 3.88 & 9.92 & 6.60 & 19.007 & 3 & 2 \\
\hline Sujetado (bra) & 4.40 & 5.04 & 8.84 & 6.96 & 36.895 & 5 & 4 \\
\hline Tanga (g-string) & 3.60 & 4.52 & 15.46 & 6.96 & 11.866 & 1 & 2 \\
\hline Tejano (jeans) & 6.24 & 3.52 & 12.76 & 6.48 & 1.700 & 2 & 3 \\
\hline Tirantes (braces) & 2.24 & 3.08 & 7.76 & 6.84 & 1.226 & 6 & 3 \\
\hline Tobillera (ankleband) & 1.20 & 1.76 & 9.84 & 6.60 & 0.987 & 0 & 4 \\
\hline Top (top) & 4.88 & 3.48 & 12.00 & 6.48 & 8.293 & 0 & 1 \\
\hline
\end{tabular}


APPENDIX (Continued)

\begin{tabular}{|c|c|c|c|c|c|c|c|}
\hline $\begin{array}{c}\text { Spanish Word } \\
\text { (English Translation) }\end{array}$ & Typ & CFam & AoA & Imag & LA & WFreq & Len \\
\hline Traje (suit) & 6.60 & 3.84 & 9.00 & 6.80 & 7.340 & 63 & 2 \\
\hline Vestido (dress) & 6.72 & 4.52 & 6.20 & 6.92 & 14.336 & 85 & 3 \\
\hline Zapatilla (gym shoes) & 3.60 & 6.52 & 5.08 & 6.84 & 23.294 & 17 & 4 \\
\hline Zapato (shoe) & 3.76 & 6.00 & 4.76 & 6.80 & 39.132 & 69 & 3 \\
\hline \multicolumn{8}{|c|}{ Intelligence } \\
\hline Abundante (abundant) & 2.76 & 5.56 & 7.92 & 4.12 & 1.399 & 23 & 4 \\
\hline Alma (soul) & 2.96 & 5.48 & 6.80 & 2.52 & 1.983 & 165 & 2 \\
\hline Amistad (friendship) & 3.48 & 6.52 & 5.34 & 3.56 & 2.348 & 53 & 3 \\
\hline Amor (love) & 3.80 & 6.52 & 5.02 & 3.96 & 2.042 & 378 & 2 \\
\hline Analizar (to analyze) & 5.52 & 4.08 & 8.76 & 2.76 & 2.113 & 25 & 4 \\
\hline Aplicado (conscientious) & 5.04 & 3.08 & 8.24 & 3.24 & 1.550 & 12 & 4 \\
\hline Aprender (to learn) & 6.04 & 6.04 & 5.18 & 3.60 & 8.345 & 27 & 3 \\
\hline Aprendiz (learner) & 5.72 & 4.92 & 10.16 & 3.28 & 3.398 & 30 & 5 \\
\hline Aprobado (passing grade) & 4.60 & 5.56 & 6.88 & 5.08 & 1.427 & 3 & 4 \\
\hline Aprobar (to pass) & 5.08 & 6.48 & 6.64 & 4.48 & 3.124 & 3 & 3 \\
\hline Atención (attention) & 5.40 & 6.44 & 5.44 & 3.28 & 1.815 & 158 & 3 \\
\hline Atento (attentive) & 5.36 & 6.16 & 5.56 & 3.88 & 1.470 & 18 & 3 \\
\hline Cabeza (head) & 5.84 & 6.60 & 4.20 & 6.72 & 5.958 & 416 & 3 \\
\hline Capacidad (capacity) & 5.92 & 6.16 & 8.13 & 3.48 & 4.713 & 114 & 4 \\
\hline Carisma (charisma) & 3.76 & 4.44 & 11.32 & 3.24 & 1.470 & 0 & 3 \\
\hline Carrera (career) & 4.48 & 6.52 & 8.84 & 4.72 & 2.268 & 77 & 3 \\
\hline Cerebro (brain) & 6.12 & 5.40 & 7.04 & 5.48 & 23.892 & 87 & 3 \\
\hline Ciencia (science) & 5.16 & 5.32 & 8.36 & 3.52 & 3.037 & 203 & 2 \\
\hline Científico (scientist) & 5.12 & 4.64 & 8.52 & 6.24 & 2.268 & 85 & 4 \\
\hline \multicolumn{8}{|l|}{ Comprender } \\
\hline (to understand) & 6.00 & 6.00 & 6.52 & 3.60 & 2.972 & 67 & 3 \\
\hline \multicolumn{8}{|l|}{ Concentración } \\
\hline (concentration) & 5.20 & 6.00 & 8.00 & 3.64 & 1.635 & 40 & 4 \\
\hline Conocer (to know) & 5.56 & 5.88 & 5.92 & 3.36 & 3.845 & 104 & 3 \\
\hline \multicolumn{8}{|l|}{ Conocimiento } \\
\hline (consciousness) & 6.08 & 5.52 & 6.84 & 3.24 & 2.265 & 203 & 5 \\
\hline \multicolumn{8}{|l|}{ Conocimientos } \\
\hline (knowledge) & 5.20 & 5.12 & 9.52 & 3.36 & 3.535 & 36 & 5 \\
\hline Copiar (to cheat) & 2.60 & 5.36 & 5.80 & 4.80 & 1.692 & 5 & 2 \\
\hline Culto (cult) & 4.96 & 5.60 & 8.12 & 4.04 & 2.574 & 30 & 2 \\
\hline Cultura (culture) & 5.36 & 6.68 & 7.48 & 4.08 & 5.333 & 165 & 3 \\
\hline Descubrir (to discover) & 5.20 & 6.12 & 6.20 & 4.00 & 1.854 & 55 & 3 \\
\hline Diez (ten) & 4.32 & 6.76 & 3.96 & 6.36 & 1.732 & 134 & 1 \\
\hline Dinero (money) & 2.72 & 6.56 & 4.04 & 6.56 & 2.845 & 199 & 3 \\
\hline Empollón (grind/nerd) & 3.92 & 3.60 & 6.56 & 5.24 & 3.461 & 0 & 3 \\
\hline Escasa (slim/limited) & 2.44 & 4.48 & 7.28 & 3.08 & 1.372 & 23 & 3 \\
\hline Escribir (to write) & 4.12 & 6.68 & 4.00 & 5.60 & 1.345 & 128 & 3 \\
\hline Espabilado (sharp) & 4.84 & 5.48 & 6.28 & 4.68 & 1.441 & 3 & 5 \\
\hline Estímulo (stimulus) & 3.48 & 5.12 & 9.28 & 3.88 & 1.680 & 16 & 4 \\
\hline Estudiante (student) & 4.52 & 5.84 & 5.96 & 6.28 & 2.454 & 19 & 4 \\
\hline Estudiar (to study) & 5.40 & 6.52 & 5.56 & 4.68 & 12.902 & 45 & 3 \\
\hline Estudio (study) & 4.84 & 6.32 & 6.00 & 5.36 & 8.888 & 144 & 3 \\
\hline Estudios (career) & 4.48 & 6.28 & 8.52 & 3.80 & 5.360 & 75 & 3 \\
\hline Estudioso (expert) & 4.80 & 4.76 & 6.80 & 4.76 & 2.717 & 3 & 4 \\
\hline Exámen (examination) & 3.76 & 5.72 & 6.64 & 6.68 & 3.246 & 21 & 3 \\
\hline Familia (family) & 2.68 & 6.28 & 3.72 & 6.52 & 1.474 & 250 & 3 \\
\hline Felicidad (happiness) & 3.44 & 5.84 & 5.64 & 3.76 & 1.652 & 85 & 4 \\
\hline Filosofía (philosophy) & 4.68 & 3.64 & 10.00 & 3.32 & 1.343 & 117 & 5 \\
\hline Futuro (future) & 3.44 & 5.60 & 7.04 & 3.24 & 2.169 & 131 & 3 \\
\hline Genio (genius) & 6.24 & 3.72 & 7.80 & 4.64 & 1.620 & 28 & 2 \\
\hline Historia (history) & 3.00 & 4.80 & 6.52 & 3.68 & 1.695 & 406 & 3 \\
\hline Hombre (man) & 4.08 & 6.24 & 4.24 & 6.68 & 2.652 & 869 & 2 \\
\hline Idea (idea) & 5.76 & 5.36 & 6.00 & 3.40 & 3.505 & 128 & 3 \\
\hline \multicolumn{8}{|l|}{ Imaginación } \\
\hline (imagination) & 5.28 & 5.20 & 5.68 & 3.56 & 4.886 & 83 & 5 \\
\hline
\end{tabular}


APPENDIX (Continued)

\begin{tabular}{|c|c|c|c|c|c|c|c|}
\hline $\begin{array}{c}\text { Spanish Word } \\
\text { (English Translation) } \\
\end{array}$ & Typ & CFam & AoA & Imag & LA & WFreq & Len \\
\hline \multicolumn{8}{|l|}{ Instituto } \\
\hline (secondary school) & 3.16 & 4.44 & 7.60 & 6.52 & 2.257 & 42 & 4 \\
\hline \multicolumn{8}{|l|}{ Inteligencia } \\
\hline (intelligence) & 7.00 & 6.00 & 8.04 & 3.24 & 2.586 & 3 & 5 \\
\hline Inteligente (intelligent) & 6.72 & 4.88 & 6.76 & 3.68 & 3.140 & 3 & 5 \\
\hline Lectura (reading) & 5.32 & 5.32 & 4.68 & 4.72 & 2.810 & 83 & 3 \\
\hline Leer (to read) & 4.76 & 6.72 & 5.12 & 5.24 & 2.482 & 97 & 2 \\
\hline Letras (letters) & 3.20 & 6.56 & 4.80 & 6.64 & 2.761 & 51 & 2 \\
\hline Libro (book) & 3.92 & 6.76 & 4.68 & 6.96 & 10.342 & 199 & 2 \\
\hline Listo (clever) & 6.20 & 5.32 & 5.20 & 3.84 & 22.067 & 16 & 2 \\
\hline Lógica (logic) & 6.16 & 4.12 & 10.28 & 3.00 & 2.795 & 74 & 3 \\
\hline \multicolumn{8}{|l|}{ Matemáticas } \\
\hline (mathematics) & 4.44 & 3.60 & 6.64 & 4.48 & 5.845 & 21 & 5 \\
\hline Memoria (memory) & 4.96 & 5.32 & 6.48 & 3.20 & 13.008 & 203 & 3 \\
\hline \multicolumn{8}{|l|}{ Memorizar } \\
\hline (to memorize) & 4.60 & 5.24 & 7.60 & 3.40 & 2.235 & 2 & 4 \\
\hline Mente (mind) & 6.24 & 4.68 & 8.20 & 2.68 & 10.509 & 87 & 2 \\
\hline Mujer (woman) & 2.92 & 6.48 & 5.24 & 6.52 & 1.626 & 511 & 2 \\
\hline NASA (NASA) & 3.48 & 2.68 & 12.16 & 4.64 & 1.616 & 1 & 2 \\
\hline Nervio (nerve) & 2.44 & 4.08 & 9.56 & 3.92 & 1.445 & 15 & 2 \\
\hline Neurona (neurone) & 5.04 & 3.08 & 11.48 & 4.16 & 5.987 & 14 & 3 \\
\hline Notas (grades) & 4.00 & 5.08 & 6.84 & 6.08 & 1.451 & 39 & 2 \\
\hline Números (numbers) & 3.76 & 5.16 & 4.76 & 6.76 & 2.725 & 28 & 3 \\
\hline Ordenador (computer) & 3.40 & 6.48 & 9.88 & 6.96 & 2.894 & 67 & 4 \\
\hline Palabras (words) & 4.28 & 6.56 & 5.40 & 6.08 & 1.538 & 354 & 3 \\
\hline Pensamieto (thought) & 5.72 & 5.56 & 8.32 & 3.28 & 14.611 & 134 & 4 \\
\hline Pensar (to think) & 6.24 & 5.76 & 7.44 & 3.08 & 11.034 & 218 & 2 \\
\hline Poder (power) & 3.32 & 4.72 & 6.96 & 2.96 & 1.903 & 388 & 2 \\
\hline Profesor (teacher) & 3.24 & 6.16 & 5.44 & 6.28 & 3.667 & 55 & 4 \\
\hline Rapidez (speed) & 5.24 & 5.24 & 6.40 & 3.44 & 3.960 & 24 & 3 \\
\hline Razón (reason) & 5.76 & 5.44 & 8.60 & 2.84 & 1.695 & 275 & 2 \\
\hline \multicolumn{8}{|l|}{ Razonamiento } \\
\hline (reasoning) & 6.60 & 5.00 & 10.32 & 2.96 & 1.602 & 8 & 5 \\
\hline Razonar (to reason) & 6.64 & 5.16 & 10.04 & 3.04 & 1.763 & 5 & 3 \\
\hline Recordar (to remember) & 4.64 & 5.12 & 7.16 & 3.32 & 2.805 & 83 & 3 \\
\hline Recuerdo (memory) & 3.52 & 5.84 & 7.40 & 4.08 & 1.418 & 177 & 3 \\
\hline Reflexión (reflection) & 5.92 & 4.64 & 9.80 & 3.16 & 1.488 & 41 & 3 \\
\hline Representación (play) & 3.48 & 3.44 & 9.72 & 3.72 & 2.235 & 46 & 5 \\
\hline Resolver (to solve) & 6.04 & 4.76 & 8.68 & 3.12 & 1.627 & 30 & 3 \\
\hline \multicolumn{8}{|l|}{ Responsabilidad } \\
\hline (responsibility) & 3.48 & 5.52 & 9.04 & 2.92 & 3.498 & 39 & 6 \\
\hline Respuesta (answer) & 4.60 & 5.28 & 6.32 & 3.44 & 1.527 & 95 & 3 \\
\hline Saber (to know) & 5.52 & 5.64 & 6.36 & 3.00 & 14.086 & 287 & 2 \\
\hline Sabiduría (wisdom) & 5.76 & 3.96 & 8.56 & 2.92 & 17.492 & 26 & 5 \\
\hline Sabio (wise) & 5.92 & 3.32 & 7.56 & 5.16 & 5.051 & 22 & 2 \\
\hline Sobresaliente (excellent) & 4.92 & 3.60 & 7.76 & 5.72 & 2.998 & 3 & 5 \\
\hline Soñar (to dream) & 3.04 & 6.04 & 5.76 & 3.92 & 2.090 & 16 & 2 \\
\hline \multicolumn{8}{|l|}{ Superdotado } \\
\hline (extremely gifted) & 5.96 & 3.12 & 9.00 & 5.12 & 4.178 & 1 & 5 \\
\hline Test (test) & 4.04 & 3.04 & 11.04 & 6.52 & 2.317 & 5 & 1 \\
\hline Tonto (fool) & 2.88 & 4.96 & 4.48 & 5.12 & 6.932 & 19 & 2 \\
\hline Trabajar (to work) & 2.92 & 5.72 & 5.56 & 4.84 & 1.714 & 74 & 3 \\
\hline Trabajo (work) & 3.08 & 5.88 & 5.56 & 5.56 & 7.890 & 354 & 3 \\
\hline Universidad (university) & 3.88 & 6.56 & 10.36 & 6.24 & 3.585 & 109 & 5 \\
\hline Vida (life) & 3.16 & 6.36 & 6.24 & 3.92 & 1.538 & 1,145 & 2 \\
\hline Voluntad (will) & 3.16 & 4.60 & 9.04 & 3.28 & 1.324 & 117 & 3 \\
\hline Yo (I) & 3.40 & 6.32 & 4.16 & 4.32 & 3.600 & 1,857 & 1 \\
\hline
\end{tabular}

(Manuscript received December 23, 2003; revision accepted for publication August 14, 2004.) 